
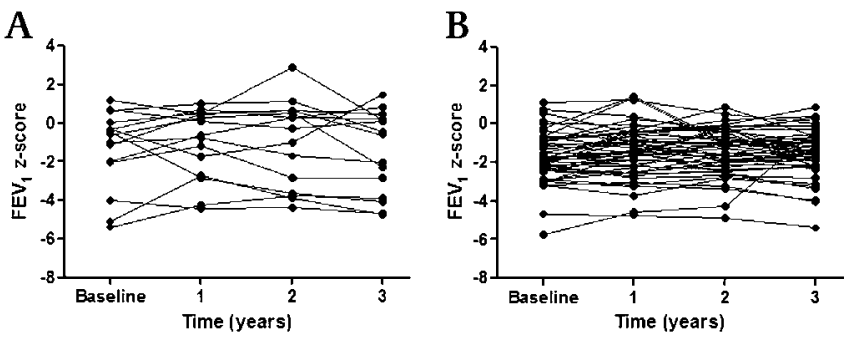

Abstract P177 Figure 1 Evolution of FEV 1 z-scores over 3 years in patients from Naples $(A, n=15)$ and from London $(B, n=50)$.

\section{Improving the investigation of suspected respiratory disease \\ P178 THE EFFECT OF BAL INDUCED INFLAMMATION ON NASAL INNATE DEFENCE - REDUCTION IN EXPERIMENTAL HUMAN PNEUMOCOCCAL CARRIAGE}

doi:10.1136/thoraxjnl-2011-201054c.178

\begin{abstract}
${ }^{1} \mathrm{~A} D$ Wright, ${ }^{2} \mathrm{~J}$ F Gritzfeld, ${ }^{3} \mathrm{~S}$ El Batrawy, ${ }^{4} \mathrm{~L}$ Roche, ${ }^{3} \mathrm{~A}$ Collins, ${ }^{2} \mathrm{~S}$ B Gordon. ${ }^{1}$ Comprehensive Local Research Network, Royal Liverpool and Broadgreen University Hospitals NHS Trust, Liverpool, UK; ${ }^{2}$ Respiratory Infection Group, Liverpool School of Tropical Medicine, Liverpool, UK; ${ }^{3} \mathrm{NIHR}$ Biomedical Research Centre, Directorate of Infection and Immunity, Royal Liverpool and Broadgreen University Hospitals NHS Trust, Liverpool, UK; ${ }^{4}$ Royal Liverpool Hospital, Cheshire and Merseyside Comprehensive Local Research Network, Liverpool, UK
\end{abstract}

Introduction and Objectives BAL causes pulmonary inflammation post procedure. It is not known if the inflammation affects the upper respiratory tract and nasal mucosa. We have developed an experimental human pneumococcal carriage (EHPC) platform. We wished to establish if prior bronchoscopy was associated with altered carriage rates in EHPC.

Methods Participants were screened for natural carriage of pneumococcus by nasal wash. Group A then proceeded to inoculation 7 days after initial screening whereas Group B underwent bronchoscopy with BAL prior to inoculation. Bronchoscopy with BAL was performed using fibre optic bronchoscope and instillation of $200 \mathrm{ml} 0.9 \%$ saline in $50 \mathrm{ml}$ aliquots followed by immediate manual aspiration via the working port of the bronchoscope. Participants were inoculated with 6B or 23F S pneumoniae (15000-60000 CFU/ $\mathrm{ml}$ ) within 14 days of bronchoscopy. Carriage was determined by the presence of pneumococci in nasal wash samples at $48 \mathrm{hr}$ and/or 7 days post inoculation.

Results Thirty-seven participants were recruited, of which 19 proceeded to BAL prior to inoculation; 22 were inoculated with $6 \mathrm{~B}$ and 15 with 23F. Baseline characteristics were not significantly different between Group A and B. Neither group had any symptoms at the time of inoculation. Both Group A and B were subdivided into $23 \mathrm{~F}$ or those that received $6 \mathrm{~B}$. The inoculum dose was not significantly different between the BAL groups for either $23 \mathrm{~F}$ or $6 \mathrm{~B}$. The mean length of time between bronchoscopy and inoculation was 10 days $( \pm 1)$. Carriage rates between Group A 6B and Group B 6B were significantly different $(p=0.008)$; this difference was not seen between Group A 23F and Group B 23F. In adults challenged with SPN, carriage rates differ by type. In an experiment with high carriage rates, there was a significant decrease in carriage rates in subjects with preceding BAL.

Conclusions This study suggests that the inflammatory process caused by bronchoscopy with BAL, as highlighted in previous research, may influence innate mucosal defence. The inflammatory effect of bronchoscopy with BAL should be accounted for in future research allowing adequate time before performing interventions which may be affected.

\section{P179 THE CHANGING NUMBERS AND INDICATIONS OF MEDIASTINOSCOPY PROCEDURES PERFORMED FOLLOWING THE INTRODUCTION OF ENDOBRONCHIAL ULTRASOUND AT A UK TERTIARY CENTRE}

doi:10.1136/thoraxjnl-2011-201054c.179

${ }^{1} \mathrm{M}$ Bahir, ${ }^{2} \mathrm{R}$ Breen, ${ }^{2} \mathrm{~A}$ Quinn, ${ }^{2} \mathrm{~J}$ King, ${ }^{1} \mathrm{G}$ Santis. ${ }^{1}$ Kings College London, London, Great Britain; 'uy's \& St Thomas' NHS Foundation Trust, London, Great Britain

Introduction Endobronchial Ultrasound(EBUS) is a minimally invasive procedure that is being increasingly utilised in the diagnosis and management of mediastinal pathologies as an alternative to surgical mediastinoscopy. This study aimed to determine the extent to which EBUS is changing the numbers of and indications for surgical mediastinoscopy at Guy's \& St Thomas' NHS Foundation Trust, a tertiary centre for EBUS and surgical mediastinoscopy.

Methods Patient records were retrospectively reviewed for two twelve-month periods, the first immediately preceding the introduction of EBUS (Phase 1), and the second commencing after a period of 15 months had elapsed (Phase 2). The numbers and indications of invasive mediastinal sampling procedures performed during each phase were determined and compared, as was the frequency of lymph node stations sampled.

Results 596 patients were included; the number of patients undergoing mediastinoscopy fell from 158 in Phase 1 to 106 in Phase 2; 332 patients underwent EBUS in Phase 2. There was significant reduction in mediastinoscopies performed to stage lung cancer $(64 \%$ reduction; $\mathrm{p}<0.001)$, confirm suspected lung cancer $(40 \%$ reduction; $\mathrm{p}<0.001)$; and diagnose granulomatous disease $(60 \%$ reduction; $p<0.001)$; however, there was a $47 \%$ increase $(p<0.001)$ in mediastinoscopies performed to diagnose mediastinal lymphadenopathy unrelated to lung cancer. In Phase 2, EBUS accounted for $81 \%$ of lung cancer staging procedures, $85 \%$ of procedures confirming suspected lung cancer, and $84 \%$ confirming granulomatous disease. Nodal stations $4 \mathrm{R} / \mathrm{L}$ and 7 were most frequently sampled by both procedures, while access to stations $10 \mathrm{R} / \mathrm{L}$ and $11 \mathrm{R} / \mathrm{L}$ by EBUS accounted for $20 \%$ of all stations sampled in Phase 2 .

Conclusion The introduction of EBUS has reduced the use of surgical mediastinoscopy, but also increased the total number of mediastinal sampling procedures performed. Mediastinoscopy use has significantly fallen for all indications that are amenable to EBUS-directed sampling.

\section{P180 ELECTROMAGNETIC NAVIGATION BRONCHOSCOPY AS A DIAGNOSTIC METHOD IN RESPIRATORY MEDICINE: EARLY CLINICAL EXPERIENCES}

doi:10.1136/thoraxinl-2011-201054c.180

S George, C Priegnitz, N Anduleit, W Randerath. Krankenhaus Bethanien, Solingen, Germany

Introduction and Objectives Electromagnetic navigation bronchoscopy (ENB) is approved for use as an adjunct to conventional bronchoscopy, aiding the diagnosis of peripheral lung lesions. It is a modern technique which improves bronchoscopic yield, thereby potentially preventing unnecessary operations or high-risk procedures. Our objective was to assess the use of this technique in regular clinical practice, and to identify factors which may influence its success.

Methods A retrospective data analysis of all ENB procedures carried out in a 120-bed speciality respiratory hospital in Solingen, Germany, between 2007 and 2011 revealed a total of 43 procedures. In each case, size and anatomical location of the tumour based on CT findings were noted. A positive result was documented if as a result of the procedure a clinical diagnosis could be reached.

Results ENB reached a clinical diagnosis in 15 of 43 patients (34.9\%); eight malignant tumours, seven benign lesions, 28 left unclear. Of these 28 , further investigations revealed a malignant process in nine 
patients, mostly by surgical interventions $(n=6) .35$ patients had previously had a failed diagnostic bronchoscopy. Of these patients, the addition of ENB allowed a diagnosis in 14 cases. 8 underwent an ENB directly upon assessment of the clinical data. The anatomical positioning of the lesion was without consequence for the diagnostic yield. The diagnostic yield increased significantly with the size of the lesion ( $<2 \mathrm{~cm}: 15 \%, 2-3 \mathrm{~cm}: 37 \%,>3 \mathrm{~cm}: 50 \%$, $p<0.001)$.

Conclusions ENB is a useful diagnostic method in the hands of a skilled interventional respiratory physician, particularly where conventional bronchoscopy has failed. Although the anatomical location does not affect the accuracy of the results, lesions over $2 \mathrm{~cm}$ in size are more likely to be amenable to this procedure. The overall diagnostic yield lies lower than those quoted in previous studies, so that selective use of this procedure should be considered.

\section{P181 COMPLICATIONS FROM CT GUIDED LUNG BIOPSIES AND RISK FACTORS FOR PNEUMOTHORAX}

\author{
doi:10.1136/thoraxjnl-2011-201054c.181
}

Z Sheikh, E Woo, T Meagher. Buckinghampshire Hopsital NHS Trust, Ayelsbury, UK

Introduction and Objectives CT guided lung biopsy is a proven diagnostic method for lung cancer. However, traditionally complication rates from this procedure have been high. The aim of our study was to assess current complication rates from this procedure and the determine what possible risk factors may account for these.

Methods A retrospective cohort of CT guided lung biopsy procedures done between August 2008 and November 2010 were analysed. Patient notes and electronic radiology records (Centricity PACS) were reviewed. Complications of haemorrhage, pneumothorax, and death were recorded and the sample adequacy of biopsy specimens for pathological examination was determined for each procedure. A univariate analysis was performed for determining the risk of post-biopsy pneumothorax and biopsy type (core or FNA), number of co-axial needle passes, needle pleural angle, lesion size, and lesion distance from pleura at point of biopsy, and lesion location (lung or mediastinal). Consequently, a multiple logistic regression analysis was performed on the most significantly correlated risk factors for pneumothorax from the univariate analysis.

Results Exactly 200 biopsy procedures done in 184 patients were included of which $64 \%$ were core biopsies $(n=128)$. The mean age was 69 years (range $31-90$ years) with with $79.5 \%$ of patients over 65 years old $(n=151)$ and $59.5 \%$ of patients male $(n=119)$. Haemorrhage occurred in $2.5 \%(n=5)$ and pneumothorax in $17 \%$ $(n=34)$ with $5 \%(n=10)$ of procedures requiring intercostals chest drain insertion for pneumothorax. No deaths were recorded. A statistically significant higher risk was observed for core biopsy (OR $3.65, \mathrm{p}=0.00,95 \%$ CI 1.38 to 9.65$)$ and lesion distance from pleura $>2 \mathrm{~cm}$ (OR 4.13, $\mathrm{p}<0.001,95 \%$ CI 1.88 to 9.08). A multivariate analysis showed that the risk was greatest when core biopsies were taken from lesions more than $2 \mathrm{~cm}$ from the pleura at point of biopsy (OR 9.14, $\mathrm{p}<0.001,95 \% \mathrm{CI} 2.72$ to 30.69). The sample adequacy rate was $95.5 \%(\mathrm{n}=191)$.

Conclusions In this recent study all complication rates were found to be lower than that reported in the national survey which is the current standard for BTS guidelines on acceptable complication rates. However, a higher rate of intercostal drain insertion was observed. Lesion distance from pleura at point of biopsy $>2 \mathrm{~cm}$ and core biopsies were the most significant risk factors for post-biopsy pneumothorax and operators should consider these prior to biopsy. Larger studies are needed to reasses current national complication rates and target complication rates may need to be specified by biopsy type.
Abstract P181 Table 1

\begin{tabular}{llllr}
\hline & & & \multicolumn{2}{l}{$\mathbf{9 5 \%}$ Cl for $\mathbf{~ O R ~}$} \\
\cline { 5 - 6 } & $\mathbf{p ~ V a l u e}$ & $\mathbf{0 R}$ & Lower & Upper \\
\hline Area = lung & 0.472 & 1.391 & 0.566 & 3.421 \\
Lesion distance from pleura $\geq 2$ & 0.001 & 3.862 & 1.800 & 8.285 \\
Lesion distance from pleura $\geq 4$ & 0.014 & 3.653 & 1.302 & 10.254 \\
Lesion size $\leq 2$ & 0.755 & 0.848 & 0.301 & 2.388 \\
Lesion size $\leq 3$ & 0.368 & 1.420 & 0.662 & 3.047 \\
More than 2 passes & 0.356 & 1.458 & 0.655 & 3.246 \\
Biopsy type $=$ core & 0.018 & 3.080 & 1.209 & 7.845 \\
Needle pleural angle $\leq 45$ & 0.757 & 0.849 & 0.302 & 2.392 \\
\hline
\end{tabular}

Abstract P181 Table 2

\begin{tabular}{lllll}
\hline & & & \multicolumn{2}{l}{$\mathbf{9 5 \%} \mathbf{C l}$ for $\mathbf{~ O R ~}$} \\
\cline { 5 - 5 } & p Value & OR & Lower & Upper \\
\hline $\begin{array}{l}\text { Lesion distance from } \\
\text { pleura } \geq 2\end{array}$ & $<0.001$ & 4.129 & 1.877 & 9.082 \\
\begin{tabular}{l} 
Biopsy type $=$ core \\
\hline
\end{tabular} & 0.009 & 3.647 & 1.384 & 9.615 \\
\hline
\end{tabular}

Abstract P181 Table 3

\begin{tabular}{lccccc}
\hline & & & \multicolumn{3}{c}{$\mathbf{9 5 \%}$ CI for OR } \\
\cline { 5 - 6 } & p Value & OR & & Lower & Upper \\
\hline Lesion distance from pleura \& biopsy type & $<0.001$ & & & \\
Lesion distance $<2$ \& type $=$ FNA & & Reference category & \\
Lesion distance $<2$ \& type $=$ core & 0.524 & 1.478 & 0.444 & 4.922 \\
Lesion distance $\geq 2$ \& type $=$ FNA & 0.941 & 0.935 & 0.159 & 5.495 \\
Lesion distance $\geq 2$ \& type $=$ core & $<0.001$ & 9.137 & 2.721 & 30.689 \\
\hline
\end{tabular}

\section{P182 UTILITY OF PET/CT REPORTING IN LUNG CANCER}

doi:10.1136/thoraxjnl-2011-201054c.182

S M Kazmi, S Sureshkumar, A Nazir, D Nazareth, J Greenwood, M Ledson, M Walshaw. Liverpool Heart and Chest Hospital, Liverpool, UK

Introduction PET scans are useful in lung cancer by facilitating accurate staging to ensure that optimal treatment can be offered. Although the European Association of Nuclear Medicine (EANM) has produced reporting guidelines, the utility of the test depends upon the interpretation of the obtained images by the reporting radiologist. Since most UK PET services are provided by the independent sector (IS) remote from cancer units, we were interested to assess the value of PET/CT reporting in the MDT management of lung cancer.

Methods We looked at the quality of reports of all 97 PET/CT scans performed in our busy lung cancer unit between December 2010 and April 2011, measuring the reports (from 6 IS radiologists) against the EANM standards. In addition, we analysed the length of the report and documentation of PET staging.

Results FDG accumulation was documented in standard guideline format (mild, moderate or intense) in 39 cases (40\%), all with SUVs noted, but in 37 cases (38\%) FDG accumulation was described as significant, increased or highgrade (36 with SUVs). However, 21 scans (22\%) had no report of FDG accumulation but 20 (90\%) still described SUVs. Corresponding CT findings were reported in 93 (96\%), and a summary report was issued in 94 (97\%), but 33 reports $(34 \%)$ were $>1$ page in length. Seven scans showed benign disease and 8 had no excess FDG activity: of the remaining 82 with malignancy, only 39 (48\%) were staged. Reasons for not staging included "inflammatory changes" (2), "uncertain findings" (18), 